\title{
Fees for uninsured services: a cross-sectional survey of Ontario family physicians
}

\author{
Jamie R. Daw PhD, Kaitlyn E. Rice BA, Danyaal Raza MD MPH
}

Abstract

Background: In Canada, family physicians are permitted to charge patient fees for administrative services that are not covered by the public health insurance program, such as prescription renewals outside of an office visit, and completion of forms and sick notes. The objective of this study was to estimate the proportion of Ontario family physicians who offer various fee structures (i.e., à la carte, annual block fees for all uninsured services rendered or no charge) for uninsured administrative services.

Methods: This was a cross-sectional telephone survey conducted from April to July 2019 of a random sample of family physicians licensed to practise in Ontario. We excluded physicians with missing contact information or additional specialties, or whose primary practice was outside of Ontario, with a walk-in clinic, with an emergency department, or with an organization that cared for a specific population (e.g., nursing home) or did not provide care (e.g., insurance company). We categorized the geographic location of practices as large urban centre (population > 100 000), small to medium centre (population 1000-99 999) or rural area. We calculated survey weights to account for nonresponse and to ensure representativeness of the sample by geographic area and payment model.

Results: Among the 221 physicians who met the inclusion criteria, the telephone was not answered at 42 practices, and the contact information was incorrect for 13, resulting in a sample of 166 physicians (response rate $75.1 \%$ ). The majority of practices reported that they charged fees for uninsured services: 97 (58.3\%, 95\% confidence interval [Cl] 50.6-65.8) charged à la carte, and 33 (20.3\%, 95\% Cl 14.8-27.3) offered patients the option to pay an annual block fee; $19(11.4 \%, 95 \% \mathrm{Cl} 7.4-17.3)$ charged no fees. Fee structures varied by geographic area but not physician payment model.

Interpretation: The use of à la carte and annual block fees for uninsured administrative services was commonly reported by a sample of Ontario family physicians. Further research is needed to examine the prevalence of patient payment of fees for uninsured services, patient and physician perceptions of fees, and concordance with regulatory guidance.

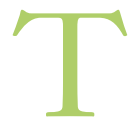
he Canada Health Act prohibits physicians from charging patient user fees for services covered by each province's public health insurance program. ${ }^{1}$ However, there is a wide range of uninsured administrative services that family physicians may provide that are not covered by public insurance, such as prescription renewals outside of an office visit, examinations for third parties such as insurers and employers, and completion of forms and sick notes. In all provinces, physicians can choose to provide these uninsured services at no cost, for a per-service charge (à la carte) or by an annual block fee that covers all uninsured administrative services for a patient or family for 1 year.

Anecdotal media reports and physician commentaries suggest that the practice of billing for uninsured services, including annual block fees, may be common among family physicians. ${ }^{2-7}$ Medicare advocacy groups have reported an increase in calls and complaints from Canadian patients about fees for uninsured services, including concerns about whether they are required to pay annual block fees and the impact of payment on access to their doctor. ${ }^{7}$ However, physicians are not required to report whether they charge à la carte or annual block fees for uninsured services, or the rates charged.

Beyond anecdotal media reports, to our knowledge, no data are available on the prevalence of physician use of fees for uninsured services in any Canadian province. The objective of this study was to estimate the proportion of family physicians who offer different fee structures for uninsured administrative services (i.e., à la carte, annual block fees or no charges) and to document variation in fee structures by geographic area and payment model.

Competing interests: Danyaal Raza is volunteer chair of the board of Canadian Doctors for Medicare. No other competing interests were declared.

This article has been peer reviewed.

Correspondence to: Jamie Daw, jrd2199@columbia.edu

CMAJ Open 2020. DOI:10.9778/cmajo.20190189 


\section{Methods}

\section{Setting}

Provincial medical associations and regulatory colleges issue detailed guidance on billing for uninsured services, and many also provide a recommended fee schedule. ${ }^{8-19}$ Table 1 lists the recommended fees for selected uninsured services in provinces with publicly available guidance on billing rates. Recommended rates are not provided for annual block fees; however, most guidance requires physicians to communicate information on annual fees, including an à la carte fee schedule, in writing to patients. In general, guidance related to fees is not legally binding. One exception is in Quebec, where physicians are required under the 2007 Health Insurance Act to post fees for uninsured services in public view and to provide patients with detailed invoices for any fees charged. ${ }^{20}$ Given the discretion offered to physicians, fee rates, patient communications and administrative procedures may vary widely. This study was conducted in Canada's largest province, Ontario, which has also been the origin of many media reports and commentaries about the rise of annual block fees. ${ }^{6,7}$

\section{Design}

To our knowledge, there is no database available with information on the use of fees for uninsured services by physicians in Canada. Thus, we conducted an observational audit study in which researchers telephoned family physician practices to ask about current fee practices for uninsured services.

We obtained contact information for 16967 family physicians licensed to practise in Ontario from the College of Physicians and Surgeons of Ontario (CPSO) public website. ${ }^{21} \mathrm{We}$ linked practice addresses to geographic areas defined by Statistics Canada (large urban centre [population $>100000$ ], small to medium centre [population 100099 999] or rural area), information about physician payment model (traditional/enhanced fee-for-service or capitationbased, obtained from the Government of Ontario website) and sociodemographic characteristics at the postal code (forward sortation area) level (obtained from the 2016 Canadian census $^{22-24}$ ).
We excluded physicians with missing contact information or additional specialties, or whose primary practice was outside of Ontario, with an emergency department, or with an organization that cared for a specific population (e.g., nursing home, travel clinic) or did not provide care (e.g., insurance company). We also excluded physicians whose primary practice was with a walk-in clinic because these physicians are less likely to have a stable patient population and thus may have a lower propensity to charge annual fees for uninsured services. From the remaining sampling frame, we drew a random sample of 275 physicians using a random-number generator.

Based on our research objectives, we developed a structured script to ask physician offices about current fee arrangements for uninsured services. We piloted the script with 10 practices and modified the script accordingly (Appendix 1, available at www.cmajopen.ca/content/8/1/E163/suppl/DC1).

A trained research assistant telephoned physician offices during normal business hours from April to July 2019). Questions were asked of whoever answered the telephone, typically an administrative staff member. If the respondent indicated that the physician offered an annual block fee, the respondent was asked to provide fee amounts, examples of the services covered and whether patient payment of the block fee conferred any other benefits (beyond uninsured services) to the patient. Two contact attempts were made for each practice. After each call, the research assistant completed a postcall evaluation form (Appendix 1).

\section{Statistical analysis}

We calculated survey weights to account for nonresponse and to ensure representativeness of the sample by geographic area and payment model. Weights were calibrated to the population distribution of the geographic and payment model variables from the full list of 16967 physicians obtained from the CPSO website. We tested variation in fee structures across physician and area-level characteristics using survey-adjusted $\chi^{2}$ tests and F-tests.

\section{Ethics approval}

This study was approved by the Columbia University Irving Medical Center Institutional Review Board.

Table 1: Recommended fees for uninsured services by province*

Province; fee, \$

\begin{tabular}{|c|c|c|c|c|c|c|}
\hline \multirow[b]{2}{*}{ Uninsured service } & \multicolumn{6}{|c|}{ Province; fee, \$ } \\
\hline & $\begin{array}{c}\text { British } \\
\text { Columbia }^{19}\end{array}$ & Saskatchewan ${ }^{10}$ & Ontario $^{12}$ & Quebec $^{14}$ & Nova Scotia ${ }^{15}$ & $\begin{array}{l}\text { Newfoundland } \\
\text { and Labrador }{ }^{17}\end{array}$ \\
\hline $\begin{array}{l}\text { Reproduction of medical records } \\
\text { ( } 20 \text { pages) }\end{array}$ & 36 & 36 & 30 & 20 & $30-75$ & 45 \\
\hline Written medical note & 45 & 80 & 19.5 & 25 & $10-20$ & $15-25$ \\
\hline Insurance medical examination & 215 & $80-640$ & 235 & 200 & $100-250$ & $130-170$ \\
\hline Prescription renewal (telephone/fax) & 32 & 80 & $\begin{array}{l}\text { Not } \\
\text { provided }\end{array}$ & 20 & $10-30$ & $15-35$ \\
\hline
\end{tabular}




\section{Results}

Of the 275 physicians in our random sample, 54 were excluded because they did not meet the inclusion criteria. Among the 221 physicians who met the inclusion criteria, the telephone was not answered at 42 practices, and the contact information was incorrect for 13 , resulting in a sample of 166 physicians (response rate $75.1 \%$ ) (Figure 1).

Of the 166 respondents, 112 (weighted prevalence $71.9 \%$ ) had a primary practice location in a large urban centre, 29 (weighted prevalence $18.7 \%$ ) in a small to medium centre, and 25 (weighted prevalence 9.4\%) in a rural area (Table 2). The majority of physicians (127 [weighted prevalence $80.3 \%$ ]) were paid through a traditional or enhanced fee-for-service model (i.e., Ontario Family Health Group); the remaining 39 physicians (weighted prevalence $19.7 \%$ ) were paid through a capitation-based model (i.e., Ontario Family Health Network or Family Health Organization).

The weighted prevalence of fee structures overall and by geographic area is shown in Figure 2. As reported by practice receptionists, 1 in 5 physicians (33 [20.3\%, 95\% confidence interval (CI) 14.8-27.3]) offered patients the option of paying an annual block fee for uninsured services, 97 (58.3\%, 95\% CI

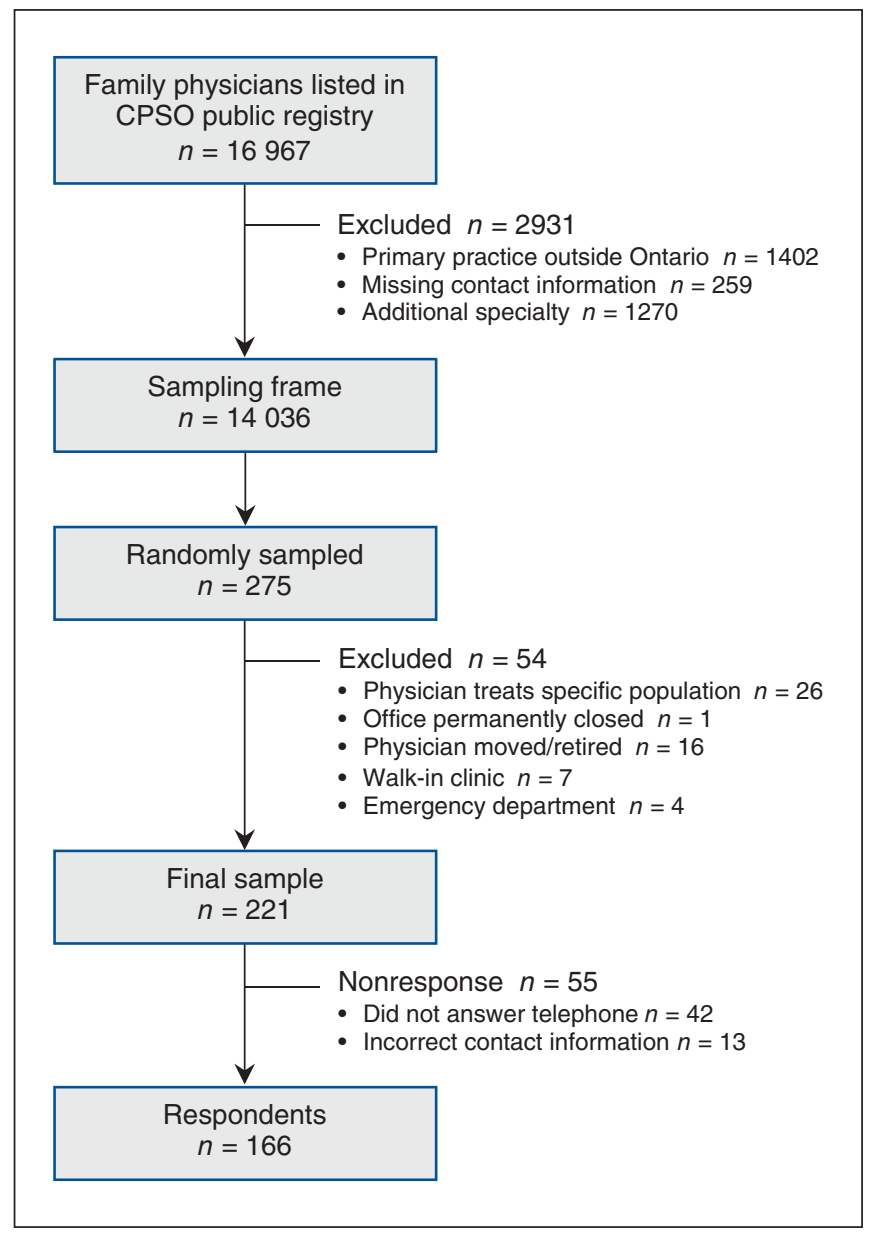

Figure 1: Flow diagram showing participant selection. Note: CPSO = College of Physicians and Surgeons of Ontario.
Table 2: Participant characteristics

\begin{tabular}{|c|c|c|c|}
\hline Characteristic & $\begin{array}{l}\text { Sample } \\
\text { size } \\
n=166\end{array}$ & $\begin{array}{l}\text { Unweighted } \\
\text { prevalence, } \\
\%\end{array}$ & $\begin{array}{l}\text { Weighted } \\
\text { prevalence, } \\
\quad \% \dagger\end{array}$ \\
\hline \multicolumn{4}{|l|}{ Geographic area* } \\
\hline Large urban & 112 & 67.5 & 71.9 \\
\hline Small/medium & 29 & 17.5 & 18.7 \\
\hline Rural & 25 & 15.1 & 9.4 \\
\hline \multicolumn{4}{|l|}{ Payment model } \\
\hline $\begin{array}{l}\text { Fee-for-service } \\
\text { (traditional or } \\
\text { enhanced) }\end{array}$ & 127 & 76.5 & 80.3 \\
\hline Capitation-based & 39 & 23.5 & 19.7 \\
\hline \multicolumn{4}{|c|}{$\begin{array}{l}\text { *Large urban centre }=\text { population greater than } 100000, \text { small/medium centre }= \\
\text { population } 1000-99999 . \\
\text { †Calculated with survey weights to account for nonresponse and to ensure } \\
\text { representativeness of the sample by geographic area and payment model. }\end{array}$} \\
\hline
\end{tabular}

50.6-65.8) charged only à la carte, and 19 (11.4\%, 95\% CI 7.417.3 ) charged no fee. All physicians in the sample who offered a block fee also gave patients the option to pay à la carte.

Fee structures did not vary significantly by physician payment model $(p=0.99)$ (Appendix 2, Supplemental Table S1, available at www.cmajopen.ca/content/8/1/E163/suppl/DC1). However, fee structures were significantly different across geographic areas $(p=0.04)$ (Figure 2). Of the 112 physicians in large urban areas, $28(25.0 \%, 95 \%$ CI $17.8-33.9)$ reported charging block fees, compared to $3 / 29(10.4 \%$, 95\% CI 3.4 $27.8)$ and $2 / 25(4.5 \%, 95 \%$ CI $1.2-15.5)$ in small to medium centres and rural areas, respectively. A lower proportion of physicians who charged block fees than of physicians who did not charge block fees practised in areas with a higher proportion of residents more than age 65 ; however, there were no differences in area-level income across fee structures (Appendix 2, Supplemental Table S2).

Among physicians who charged block fees, the mean annual amount was \$106 (95\% CI \$92-\$121) for an individual and \$194 (95\% CI \$171-\$217) for a family (Figure 3; Appendix 2, Supplemental Table S3). The most commonly reported services covered by the fee were form completion, doctor's notes and prescription renewals (by telephone or email). All 33 practices that charged a block fee reported that payment would not provide additional benefits, such as improved access to the physician, to the patient.

\section{Interpretation}

In our sample, the majority of Ontario family physicians indicated that they charged patients à la carte for uninsured administrative services, and 1 in 5 offered patients the option to pay an annual block fee for all uninsured administrative services rendered at their practice. Given estimates of 13340 primary care physicians (based on 2015/16 data) and a mean primary care practice size of 1888 (based on 2010/11 data), about 5 million patients in Ontario may be receiving letters from their family physician about annual block fees each year. ${ }^{25,26}$ 


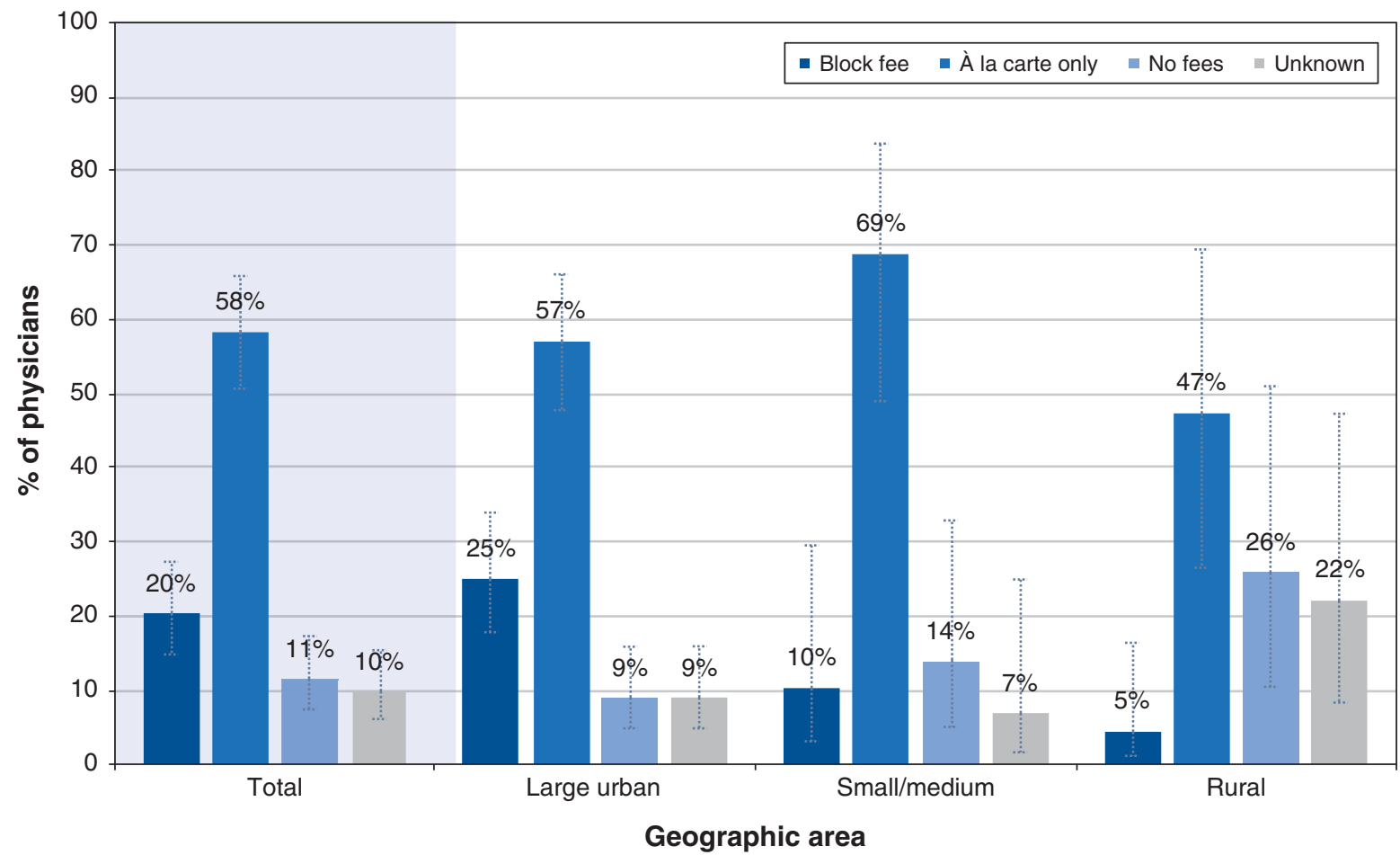

Figure 2: Weighted prevalence of family physician fees for uninsured administrative services, total and by geographic area. All physicians who offered a block fee also gave patients the option to pay à la carte. Large urban centre $=$ population greater than 100000 , small/medium centre $=$ population 1000-99 999. Error bars represent 95\% confidence intervals.
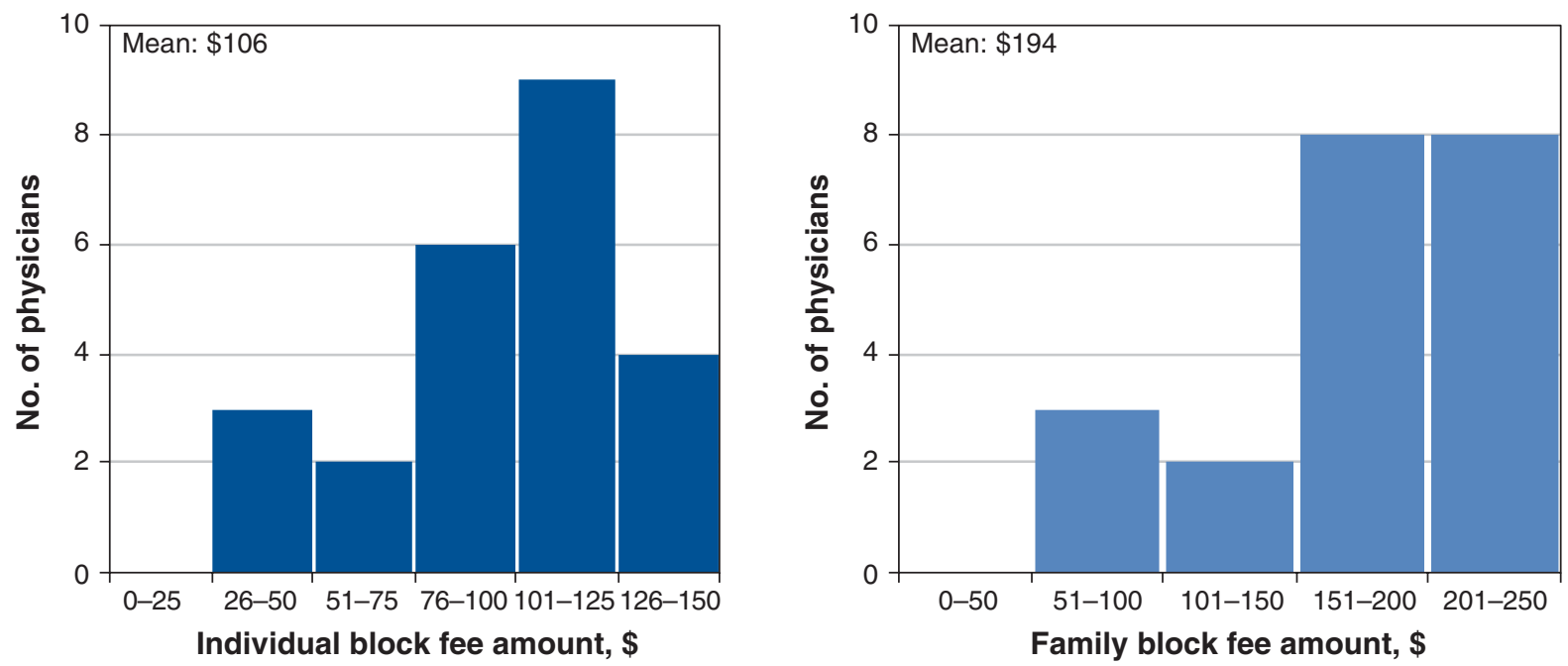

Figure 3: Distribution of family physician annual block fees for uninsured administrative services. 
The geographic characteristics of the sample are consistent with other descriptive data for Ontario's primary care workforce: a 2013 analysis showed that $9.3 \%$ of general practice and family physicians work in rural areas of the province, ${ }^{27}$ virtually the same as our weighted prevalence of $9.4 \%$. However, our sample had a lower proportion of practices that reported capitation-based payment than that documented in other reports. An analysis of Ontario physician compensation from 2015-2016 showed that $38 \%$ of family physicians were enrolled in a capitation-based payment model, ${ }^{25}$ compared to $19.7 \%$ in our sample.

Our findings suggest that physician fees for uninsured services are common in Ontario and that more research into the details of their use, the prevalence of payment by patients and the potential impact on patients is warranted.

The CPSO policy emphasizes the need to mitigate the impact of these charges on patients, stating that doctors "must ensure that patient decisions regarding whether to pay a block fee do not affect their ability or the ability of other patients in the practice to access health-care services." ${ }^{13}$ It also requires physicians to charge "reasonable" fees (whether à la carte or annual fees) and to provide patients with written information on block fee options for uninsured services. ${ }^{13}$ The CPSO policy explicitly recognizes that confusion could arise when patients pay for uninsured services and also notes that physicians need to be clear and impartial when proposing uninsured services as an alternative or in combination with insured services. ${ }^{13}$ However, media reports have included examples of physician letters to patients about block fees that have used aggressive, confusing and political language, including making connections between spending cuts by provincial governments and the need to charge an annual fee. ${ }^{28}$ Future research should examine communication practices around patient fees, particularly annual block fees, and how they affect patients' perceptions of the purpose and implications of fee payment.

Finally, more information is needed on the uninsured services for which physicians most commonly charge patient fees and the concordance of billing rates with regulatory guidance. These data could assist provincial governments in considering whether public coverage is warranted for some uninsured services, particularly those related directly to necessary medical care, such as prescription renewals outside of an office visit. The total sum of payments for uninsured services is likely small: all out-of-pocket expenditures for physician services accounted for only $1.7 \%$ of total physician expenditures in Canada in 2016. ${ }^{29}$ However, the average individual block fee of $\$ 106$ in the current study is relatively large compared to per capita out-of-pocket spending on prescription drugs ( $\$ 188$ in 2018) and total per capita out-of-pocket spending on health care (\$972 in 2016). ${ }^{29,30}$

\section{Limitations}

This study has several limitations that should be considered when interpreting the results. First, we did not capture physician or patient perceptions of patient fees or their impact on patient access to health care.
Second, we estimated only the prevalence of use of fees as reported by physician practices, not by physicians, and not the prevalence of fees paid by patients. To our knowledge, there is no available source of data to validate whether (and how often) individual physicians actually charge fees to their patients or how often these fees are paid. It is possible that relatively few patients are actually charged à la carte fees or that few patients opt in to the annual block fee when it is offered. The CPSO policy states that physicians must consider a patient's ability to pay fees and "allow for flexibility on compassionate grounds." 13

Third, since we used a public registry to identify physicians, limited information was available to explore variation in fees across physician characteristics.

Fourth, the sample was small, and we did not conduct an a priori sample size calculation. Assuming a population prevalence of $50 \%$ (the most conservative assumption), the sample size of 166 physicians allowed us to estimate the proportion of practices that charge fees for uninsured services with a margin of error of $7.6 \%$. Assuming a standard deviation of $\$ 100$, this sample size allowed us to estimate the mean block fee rate with a margin of error of $\$ 15$.

Fifth, the findings of this study may not be generalizable to other provinces; more research is needed to explore variation in uninsured fee practices across Canada.

Finally, we identified a lower proportion of physicians enrolled in a capitation-based payment model than did other investigators. ${ }^{25}$ This likely reflects the low specificity of the method used to allocate physicians to capitation-based models based on information on the Government of Ontario website (which may not be complete or updated). As a result, our comparisons of fee prevalence across payment models are likely attenuated.

\section{Conclusion}

Our findings suggest that further investigation into the prevalence, perception and administration of fees for uninsured services is warranted. To improve the data available on patient fee practices, professional bodies and provincial governments could require physicians to report uninsured administrative fee schedules or provide copies of patient letters regarding annual block fees, or both. A public reporting mechanism could also be established. These data would assist with monitoring of compliance with regulatory college policies and inform the need for, and development of, further physician guidance and enforcement mechanisms.

\section{References}

1. Canada Health Act (R.S.C., 1985, c.C-6). Available: https://laws-lois.justice. gc.ca/eng/acts/C-6/page-1.html (accessed 2019 Oct. 15).

2. Glauser W. Annual fees: Survival or cash grab? CMA7 2011;183:781-2.

3. Ladouceur R. Extra fees for uninsured services. Can Fam Physician 2016;62:373.

4. Priest L. Can my doctor charge me an annual fee? Globe and Mail [Toronto]. Available: www.theglobeandmail.com/life/health-and-fitness/ask-a-health -expert/can-my-doctor-charge-me-an-annual-fee/article595289/ (accessed 2019 Oct. 15).

5. Can my doctor charge me for refilling a prescription? Globe and Mail [Toronto]. Available: www.theglobeandmail.com/life/health-and-fitness/ask-a -health-expert/can-my-doctor-charge-me-for-refilling-a-prescription/article 579941/ (accessed 2019 Oct. 15). 
6. Wijayasinghe S. My doctor wants to charge me an annual fee. Can I opt out? Globe and Mail [Toronto]. Available: www.theglobeandmail.com/life/health -and-fitness/ask-a-health-expert/my-doctor-wants-to-charge-me-an-annual-fee -can-i-opt-out/article14217831/ (accessed 2019 Oct. 15).

7. Collier R. Aggressive billing techniques confusing Canadians. CMA7 2008;179: 888-9.

8. Charging for uninsured services [practice standard]. Vancouver: College of Physicians and Surgeons of British Columbia; 2019. Available: www.cpsbc.ca/ files/pdf/PSG-Charging-for-Uninsured-Services.pdf (accessed 2019 Oct. 15).

9. Charging for uninsured professional services. Edmonton: College of Physicians and Surgeons of Alberta; 2010, reissued 2014 Sept. 9. Available: http://www.cpsa. $\mathrm{ca} /$ standardspractice/charging-for-uninsured-professional-services/ (accessed 2019 Oct. 15).

10. SMA fee guide (uninsured services). Saskatoon: Saskatchewan Medical Association (SMA); 2018. Available: https://www.sma.sk.ca/kaizen/content/files/_SMA\%20 Fee\%20Guide\%20October\%201,\%202018\%20(updated\%20Feb\%202019).pdf (accessed 2020 Mar. 2).

11. Standards of practice of medicine. College of Physicians and Surgeons of Manitoba; 2019. Available: www.cpsm.mb.ca/laws-and-policies/standards-of -practice-of-medicine. (accessed 2019 Oct. 16).

12. Physician's guide to uninsured services: a guide for Ontario physicians. Toronto: Ontario Medical Association; 2019:1-52. Available: http://swpca.ca/Uploads/ ContentDocuments/2019-Physicians-Guide-to-Uninsured-Services-10Jan19\% 20(004).pdf (accessed 2019 July 22).

13. Uninsured services: billing and block fees. Toronto: College of Physicians and Surgeons of Ontario; updated 2000. Available: www.cpso.on.ca/Physicians/ Policies-Guidance/Policies/Uninsured-Services-Billing-and-Block-Fees (accessed 2019 June 30).

14. Proposed provincial fees for non insured services and incidental fees provided by family physicians. Westmount (QC): Fédération des médecins omnipraticiens du Québec; 2019. Available: http://fmoq.s3.amazonaws.com/pratique/ facturation/frais-accessoires/Grille-tarifaire-FMOQ-032019_EN.pdf (accessed 2019 Oct. 17)

15. Physicians' guide to billing for non-insured services. Dartmouth (NS): Doctors Nova Scotia; 2018. Available: https://doctorsns.com/sites/default/files/2018-04/ PhysiciansGuideToBillingForNonInsuredServices.pdf (accessed 2019 Oct. 15).

16. Charging for uninsured services. Rothesay (NB): College of Physicians and Surgeons of New Brunswick. Available: https://cpsnb.org/en/medical-act -regulations-and-guidelines/guidelines/439-charging-for-uninsured-services (accessed 2019 Oct. 16)

17. Physicians' guide to non-insured services. St. John's: Newfoundland and Labrador Medical Association; 2018. Available: www.nlma.nl.ca/FileManager/ Publications/Guides/nlma-physicians-guide-to-non-insured-service-june-2018. pdf (accessed 2019 Oct. 16).

18. Charging for uninsured services. Charlottetown: The College of Physicians and Surgeons of Prince Edward Island; 2017. Available: http://cpspei.ca/wp -content/uploads/2017/03/Charging-for-Uninsured-Services-Sept-913.pdf (accessed 2019 Oct. 16).

19. Revised fees for uninsured services, effective April 1, 2019. Vancouver: British Columbia Medical Association. Available: https://www.doctorsofbc.ca/sites/ default/files/public_uninsured_services1apr2019.pdf (accessed 2020 Mar. 2).
20. Chapter A-29: Health Insurance Act. Québec: Les Publications du Québec; updated 2019 Dec. 10. Available: http://legisquebec.gouv.qc.ca/en/ShowDoc/ cs/A-29 (accessed 2019 Oct. 17).

21. Find a doctor. Toronto: College of Physicians and Surgeons of Ontario. Available: https://doctors.cpso.on.ca/?search=general (accessed 2019 Feb. 5).

22. Population centre and rural area classification 2016. Ottawa: Statistics Canada; modified 2017 Feb. 8. Available: www.statcan.gc.ca/eng/subjects/standard/ pcrac/2016/introduction (accessed 2019 Feb. 15).

23. Primary care payment models in Ontario. Toronto: Ontario Ministry of Health and Long-Term Care; 2019. Available: www.health.gov.on.ca/en/pro/ programs/pcpm/ (accessed 2019 Feb. 5).

24. Census profile, 2016 census. Ottawa: Statistics Canada; modified 2019 July 17. Available: www12.statcan.gc.ca/census-recensement/2016/dp-pd/prof/index. cfm?Lang=E (accessed 2019 July 22).

25. Schutz S, Glazier R, Graves E, et al. Payments to Ontario physicians from Ministry of Health and Long-Term Care sources: update 2005/06 to 2017/18. Toronto: ICES; 2019. Report no 0866010 004. Available: www.ices.on.ca/Publications/ Atlases-and-Reports/2012/Payments-to-Ontario-Physicians (accessed 2020 Jan. 31).

26. McLeod L, Buckley G, Sweetman A. Ontario primary care models: a descriptive study. CMA7 Open 2016;4:E679-88.

27. Wenghofer EF, Kam SM, Timony PE, et al. Geographic variation in FP and GP scope of practice in Ontario. Can Fam Physician 2018;64:e274-82.

28. Csanady A. Paying to be a patient? Toronto woman calls doctor's annual $\$ 150$ fee request 'galling.' National Post [Toronto]. Available: https:// nationalpost.com/news/politics/paying-to-be-a-patient-toronto-woman-calls -doctors-annual-150-fee-request-galling (accessed 2019 Oct. 15).

29. National bealth expenditure trends, 1975 to 2018. Ottawa: Canadian Institute for Health Information; 2019. Available: https://secure.cihi.ca/free_products/nhex -trends-narrative-report-2019-en-web.pdf (accessed 2019 Oct. 16).

30. Drug spending at a glance. Ottawa: Canadian Institute for Health Information 2018. Available: www.cihi.ca/sites/default/files/rot/rot-document/nhex-drug -infosheet-2018-en-web.pdf (accessed 2019 Oct. 16).

Affiliations: Department of Health Policy and Management (Daw, Rice), Columbia University Mailman School of Public Health, New York, NY; Department of Family and Community Medicine (Raza), Faculty of Medicine, University of Toronto; Department of Family and Community Medicine (Raza), St. Michael's Hospital, Toronto, Ont.

Contributors: Jamie Daw conceived the study. Jamie Daw, Danyaal Raza and Kaitlyn Rice contributed to the study design. Jamie Daw and Kaitlyn Rice collected and analyzed the data. Jamie Daw drafted the manuscript, and Danyaal Raza and Kaitlyn Rice revised it critically for important intellectual content. All of the authors approved the final version to be published and agreed to be accountable for all aspects of the work.

Supplemental information: For reviewer comments and the original submission of this manuscript, please see www.cmajopen.ca/content/8/1/ E163/suppl/DC1. 